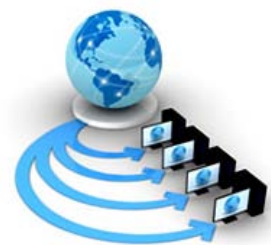

Volume 9, no. 2, march-april 2018

International Journal of Advanced Research in Computer Science

RESEARCH PAPER

\author{
Available Online at www.ijarcs.info
}

\title{
ANALYSIS OF MRI IMAGES USING DATA MINING FOR DETECTION OF BRAIN TUMOR
}

\author{
Sameena Naaz \\ Department of Computer Science and Engineering, \\ School of Engineering Sciences and Technology, Jamia \\ Hamdard, New Delhi-110062, India
}

\author{
Hema Malhotra \\ Department of Computer Science and Engineering, \\ School of Engineering Sciences and Technology, Jamia \\ Hamdard, New Delhi-110062, India
}

\begin{abstract}
Brain tumor is a formation of abnormal cells within the brain that can disrupt the function of the brain. In India, every year 40,00050,000 persons are diagnosed with brain tumor. Traditionally, radiologist manually detect and calculate the size of the tumor from CT Scan images during regular screening. Out of which, approximately, $10 \%$ to $30 \%$ of tumors are missed by them. In this paper, a computer aided system for brain tumor detection and it's area calculation using $\mathrm{K}$ - means clustering algorithm has been proposed using an interface.
\end{abstract}

Keywords: Brain Tumor, Clustering, Image Segmentation, K-means algorithm, Median filter, MRI.

\section{INTRODUCTION}

Magnetic resonance imaging (MRI) is a type of scan that uses magnetic fields and radio waves, rather than X-rays, and computers to create detailed pictures of the brain. Magnetic resonance imaging (MRI) is the most common types of tests used to diagnose brain tumors. It uses computers to create detailed images of the brain. The best type of imaging to diagnose most types of brain tumors is MRI. The nucleus of an atom contains protons and neutrons and its angular momentum can be defined as a spin. When the number of subatomic particles in the nucleus is even, it results in the cancellation of the spin. And, only odd number of subatomic particles in the Nuclei will have a resultant spin.This forms the basis of magnetic resonance imaging.

A Brain tumor is a formation of abnormal cells within the brain that can disrupt the function of the brain. A Brain tumor is a formation of abnormal cells within the brain that can disrupt the function of the brain. Some common types of brain tumor are Oligodendroglioma, glioblastoma, Gliomas and Eningiomas [1].

When our body is functioning in a normal manner, the cells keep on dying and getting replaced by new cells. But this normal cycle gets disrupted in tumors and cancer. The tumor cells keep on growing and do not die out even though our body does not need them. This uncontrolled growth of cells results in improper functioning of our body part, brain in our case. The growth of these cells in the brain could be mass or malignant. In this work a Graphical user Interface has been created for easy input of MRI images into the system for further processing. The work has been carried out in MATLAB and it focuses on detection of mass of benign tumor. The system developed helps in detecting the presence or absence of tumor in an MRI image and also gives the area of brain affected by the disease.

An image can be represented as a matrix or a rectangular array consisting of rows and columns. The first step is to collect the image which is reffered to as image acquisition or imaging. This image consists of a number of picture elements or pixels. The pixel at every location has some value associated with it. The next step is to pre-process the image to enhance it for further processing. This is done by changing the nature of image to enhance its pictorial information for better human interpretation.

Segmentation is a very essential process and holds a critical position in the field of image processing to extract information from complex medical images. The main objective of the image segmentation is to partition the digital image into mutually exclusive regions. Image segmentation can be carried out in a number of ways. Some of these are Region based segmentation, Edge detection segmentation, segmentation based on clustering and segmentation based on weakly supervised learning in CNN. Although these algorithms give good results but we need to have fast and more efficient techniques for medical image segmentation [2].

In our work we have done segmentation using clustering. A cluster is a group of objects which have similar properties, but are different from the objects of another cluster. This is a method of unsupervised learning finds structure in a collection of unlabelled data in order to categorize it into various clusters. Several algorithms exist for clustering and the one used in our work is K-means clustering. This algorithm groups objects into $\mathrm{k}$ number of clusters/groups based on features/attributes where $\mathrm{k}$ is some random positive integer. 


\section{RELATED WORK}

Tumor may be defined as Mass i.e, in the brain or as Malignant i.e, over the brain. If it is defined as mass then Kmeans algorithm is sufficient to extract the tumor from the brain cells. The MRI images have noise which could be due to RF pulses, field strength, RF coil, receiver bandwidth or voxel volume. This noise could be removed using various techniques such as Markov random field, Anisotropic diffusion, Wavelet-based method, Bilateral and Trilateral filtering etc.

This noise free image is the input to the K-means segmentation process, which uses approximate reasoning, for calculating shape and position of the tumor [3].

Image processing can be defined as an active research area where MR image processing is a very challenging field. Medical imaging technologies can be used for imaging different parts of the human body for medical diagnosis. Brain tumor is a very serious and life-threatening disease. Image segmentation plays an important role in image processing as it is used in the extraction of suspicious parts from the MRI (magnetic resonance imaging) images. The paper proposed segmentation of the human brain MRI image using K-means clustering algorithm followed by the morphological filtering which is used to avoid the misclustered regions formed after segmentation[4].

The purpose of the paper [5] was to provide the review for the various MRI based brain tumor segmentation methods. Firstly, space a brief introduction to brain tumors and imaging modalities. Then, proceeding with the comparison in different imaging modalities. Finally, the brief discussion of the current state is performed and the qualities of different approaches are critically reviewed.

[6] proposed automatic brain tumor detection approach using symmetry analysis. The sequence of the methodology proposed in this paper shows the detection of tumor at the beginning and then segmenting the area of interest followed by area of tumor calculation. One of the important aspects after performing the quantitative analysis is that we can identify the status of the disease. Modular multi-step approach is used in the paper for solving complex MRI segmentation problem Their algorithm can be used to automatically detect and segment the brain tumor. Here, the author agrees that MR imaging can give better results as compared to the other techniques like CT images and Xrays. To remove noise, Pre-processing of the image is done by converting the RGB image into gray-scale image and then passing the same image to the high pass filter.

\section{Proposed Method}

In this paper, a user-friendly computer aided system for brain MRI image segmentation for detection of tumor as well as it's area calculation using $\mathrm{K}$ - means clustering algorithm has been proposed using an interface of GUI in MATLAB. The block diagram is shown and each block is described in Figure 1.

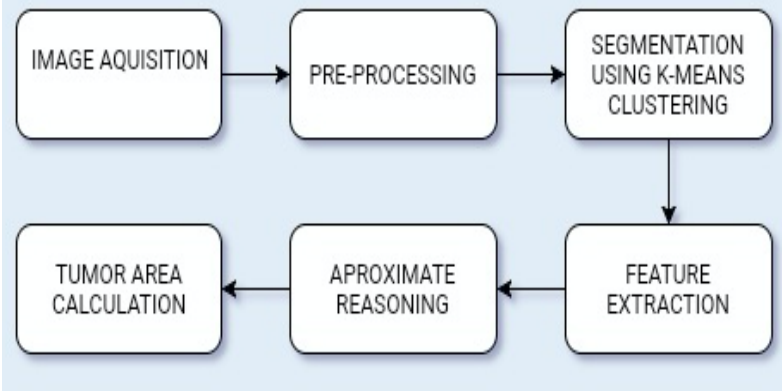

Figure 1. Block Diagram

\subsection{Image Acquisition}

Here, Images are obtained using MRI scan of the human brain where the gray-scale images are given as the input. The range of values for gray-scale image is from 0 (black) to 255 (white).

\subsection{Pre-Processing}

This step helps in improving the quality of the image, making it more suitable for further processing. Here, we convert the RGB input image to gray-scale. It also includes steps like Image enhancement, contrast improvement and image sharpening.

\subsubsection{Enhancement of MRI Images}

Here, enhancement techniques are used to improve the image information for human views to obtain accurate outcomes. Here, only the brightness of the images is increased in order to enhance the visibility of the image which was done to improve the quality of the brain MRI images.

\subsubsection{Contrast Improvement}

MRI images also called RGB images are converted into gray scale images (intensity images). Here, a MATLAB function 'imadjust' has been used which increases the contrast of an image by mapping the values of the input intensity image to new values such that the data is saturated at low and high intensities of the input data.

\subsubsection{Noise Removal}

There can be significant amount of noise in MRI images which could be due to environment, equipment or operator carelessness. These could lead to major inaccuracies in MRI. So, the first step is to remove the noise present in the MRI image. The methods used for noise reduction can be linear or non-linear. In Linear filters, for noise reduction, the pixel value is updated by weighted average of neighbourhood. This method reduces the image quality. On the other hand in non-linear method, the edges are pre- 
served but the fine structures are degraded.

Here, we are using a median filter. The median filter is a nonlinear digital filtering technique, often used to remove noise from an image or signal.

\subsubsection{Sharpening of image}

After this high pass filter is used to sharpen the image. There is a need to sharpen the edges for proper detection of boundary of tumor. Very good results are obtained from high pass filter and so it is used for enhancing the details of the image.

\subsection{K Means Clustering for Segmentation}

After pre-processing the image is segmented using $\mathrm{K}$ Means clustering. Segmentation algorithms are area oriented instead of pixel oriented. Segmentation is concerned with dividing an image into meaningful regions and the result of segmentation is the splitting up of the image into connected areas [7]. The main objective of the image segmentation is to partition the digital image into multiple segments.

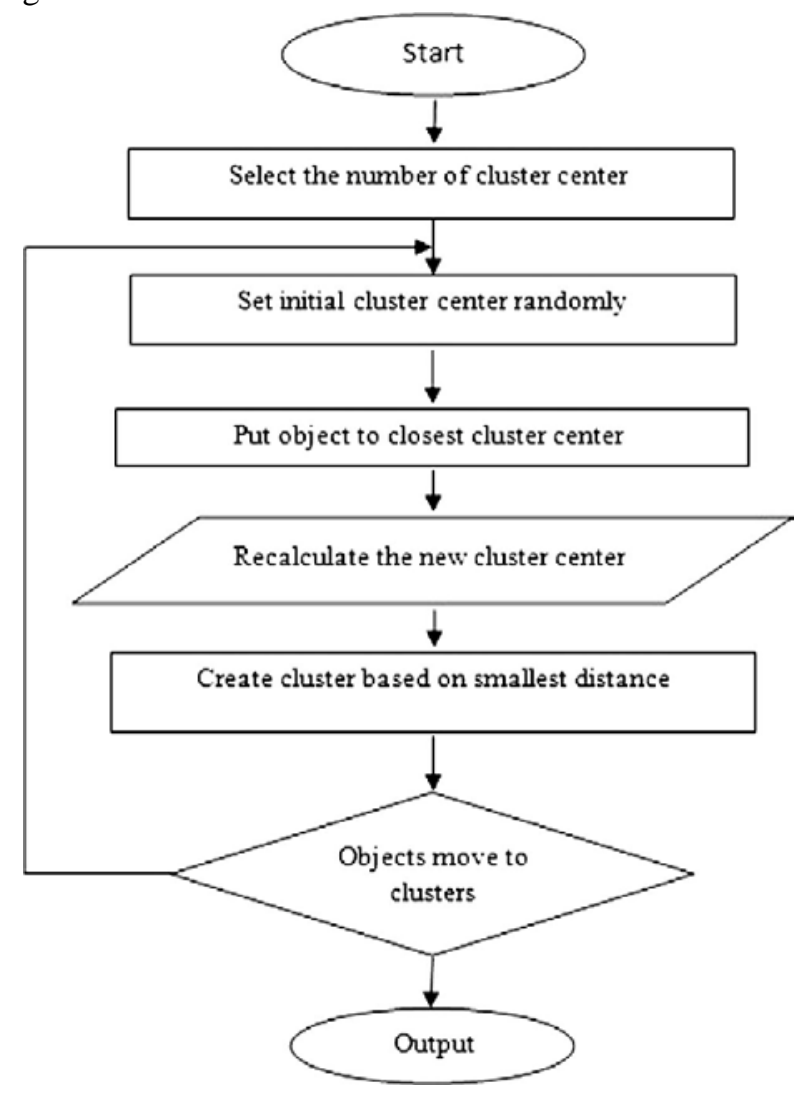

Figure 2. Flowchart for Segmentation using Clustering

Accurate segmentation is a crucial step in medical imaging analysis and classification. There are many challenges associated in performing the brain MR image segmentation [8].

Segmentation is done using clustering. The classification of similar objects or the partitioning of dataset into clusters such that each cluster share some common trait is known as clustering [9]. Clustering is used to group unleveled data. Here, K-Means is used to cluster the intensity value of the image and thus the image is segmented into different regions. In $\mathrm{k}$-means, $\mathrm{k}$ points representing the initial group of centroids are placed into space which are represented by the objects that are being clustered. Then, each object is assigned to the group that has the closest centroid[10]. Using K-Means, an index image can be obtained which has four clusters and then every cluster is segmented in a different image. And, then the cluster containing the tumor is selected. Figure 2 shows the flowchart representation of the $\mathrm{K}-\mathrm{Means}$ algorithm and the results are given in figure 3 .

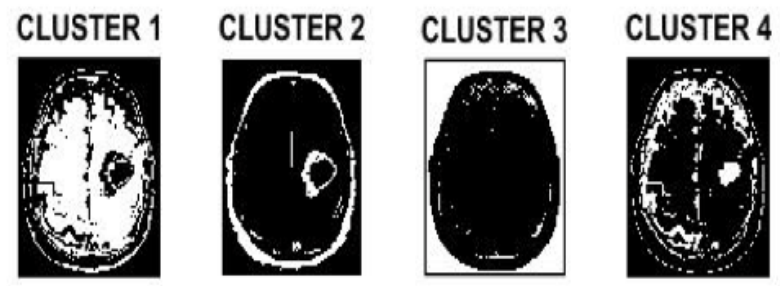

Figure 3. Results of K-Means Clustering

\subsection{Feature Extraction}

Thresholding is used for feature extraction, which involves the extraction of the cluster showing the predicted tumor at the output of k-means. After this threshold processing of the extracted cluster is done.

Feature extraction is done by thresholding. The feature extraction process involves extracting the cluster, which shows the predicted tumor at the output of k-means. The extracted cluster is then given for the threshold processing.

\subsection{Approximate Reasoning}

The shape and position of the tumor is finally recognized used approximate reasoning. In the approximate reasoning step, a binary mask is applied over the entire image resulting in the dark pixel becoming dark and and white pixel becoming brighter.

\subsection{Tumor Area Calculation}

Number of white pixels (0) in the binary image is used to calculate the tumor area. Binarization method is used for this calculation. The image taken here is a binary image, so it has only black and white pixels and the maximum size is 256X256. A value 0 is assigned to black pixel and a value 1 is assigned to white pixel.

$\mathrm{f}(1)=$ white pixel (assigned as 1)

$\mathrm{f}(0)$ = black pixel (assigned as 0 )

The area calculation formula is as follows: 
Size of tumor is $\mathrm{S}=\left[(\sqrt{\mathrm{P}})^{*} 0.264\right] \mathrm{mm}^{2}$

Where, $\mathrm{P}=$ number of white pixels and value of 1 Pixel $=$ $0.264 \mathrm{~mm}$.

\section{RESULTS}

The brain tumor can be found by using a computer aided system using an interface of GUI in Matlab. A GUI (Graphical User Interface) is created to make the system userfriendly.

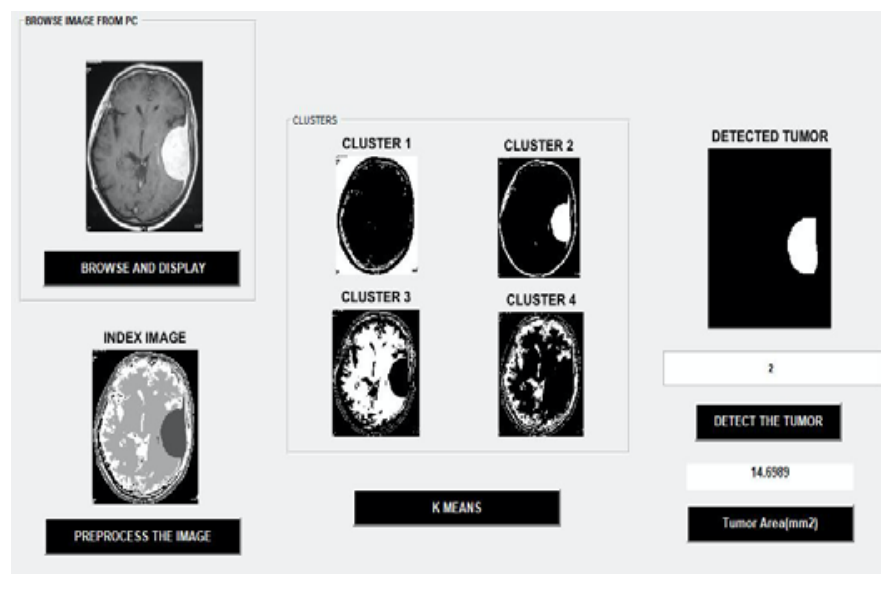

Figure 5. Results

\section{CONCLUSION}

In this project, a computer aided system for brain tumor detection and it's area calculation using $\mathrm{K}$ - means clustering algorithm has been proposed using an interface of GUI in Matlab. The GUI developed in this work provides better interface and visualization as compared to traditional methods. Faster input output and manipulation of parameters is possible which results in faster and more efficient method for detection of tumor. The results are clearly more accurate and faster and we were able to detect the tumor and calculate it's area for different brain MRI images from our database.

\section{REFERENCES}

[1] Sharma, K., Kaur, A., \& Gujral, S. (2014). A review on various brain tumor detection techniques in brain MRI images. IOSR Journal of Engineering, 4(5), 6-12.

[2] Abdel-Maksoud, E., Elmogy, M., \& Al-Awadi, R. (2015). Brain tumor segmentation based on a hybrid clustering technique. Egyptian Informatics Journal, 16(1), 71-81.

[3]. Lakshmi, A., \& Arivoli, T. (2015). Brain Tumor Segmentation and its Area Calculation in Brain MR Images using K-Mean Clustering and Fuzzy C-Mean Algorithm.

[4]. Joseph, R. P., Singh, C. S., \& Manikandan, M. (2014). Brain tumor MRI image segmentation and detection in image processing. International Journal of Research in Engineering and Technology, 3(1), 1-5.

[5]. Khurana, S., \& Garg, M. L. (2015). MRI based Brain Tumor Segmentation Methods: A Critical. International Journal, 3(4).

[6] Roy, S., Nag, S., Bandyopadhyay, S. K., Bhattacharyya, D., \& Kim, T. H. (2015). Automated brain hemorrhage lesion segmentation and classification from MR image using an innovative composite method. Journal of Theoretical and Applied Information Technology, 78(1), 34.

[7] Saini, P. K., \& Singh, M. (2015). Brain Tumor Detection in Medical Imaging Using Matlab. International Research Journal of Engineering and Technology, 2(02), 191-196.

[8] Vani, V. (2015). Review on Automated Brain Tumor Segmentation and classification from Brain MRI. International Journal of Advanced Scientific and Technical Research, 2, 2249-9954.

[9] Ghosh, S., \& Dubey, S. K. (2013). Comparative analysis of k-means and fuzzy c-means algorithms. International Journal of Advanced Computer Science and Applications, 4(4), 35-39.

[10] Patil, M. (2013). Mrs. Prachi Kshirsagar, Samata Prabhu, Sonal Patil, Sunilka Patil,” Brain Tumor Identification Using K-Means Clustering”. International Journal of Engineering Trends and Technology, 4(3), 354-357. 